\title{
LncRNA LINC00342 contributes to the growth and metastasis of colorectal cancer via targeting miR-19a-3p/NPEPL1 axis
}

\author{
Peng Shen ${ }^{1}$, Lili Qu' ${ }^{1}$ Jingjing Wang ${ }^{1}$, Quchen Ding ${ }^{1}$, Chuanwen Zhou², Rui Xie², Honggang Wang ${ }^{2}$ \\ and Guozhong $\mathrm{ji}^{1 *}$ (i)
}

\begin{abstract}
Background: Long intergenic non-protein coding RNA 00342 (LINC00342) has been identified as a novel oncogene. However, the functional role of LINC00342 in colorectal cancer (CRC) remains unclear.

Methods: The expression of LINC00342 is detected by real-time PCR (RT-PCR) analysis. Cell proliferation, migration and invasion and xenograft model are examined to analyze the biological functions of LINC00342 in vitro and in vivo using colony formation, would healing and transwell analyses. Dual-luciferase reporter and RNA immunoprecipitation (RIP) assays are used to identify the target interactions between LINC00342, miR-19a-3p and aminopeptidase like 1 (NPEPL1).

Results: LINC00342 was highly expressed in CRC. Down-regulation of LINC00342 inhibited cell proliferation and metastasis of CRC cells. Moreover, knocking down LINC00342 inhibited the tumor growth in vivo. Mechanistic investigation revealed that LINC00342 might sponge miR-19a-3p to regulate NPEPL1 expression. Further investigation indicated that the ontogenesis facilitated by LINC00342 was inhibited due to the depletion of NPEPL1.
\end{abstract}

Conclusion: LINC00342 promotes CRC progression by competitively binding miR-19a-3p with NPEPL1.

Keywords: Colorectal cancer, Invasion, LINC00342, Proliferation, miR-19a-3p, NPEPL1

\section{Highlights}

1. LINC00342 is highly expressed in CRC tissues and cells.

2. Knockdown of LINC00342 inhibits CRC cell proliferation, migration and invasion in vitro.

3. LINC00342 acts as a ceRNA for miR-19a-3p to regulate NPEPL1 expression.

*Correspondence: jgz@njmu.edu.cn

${ }^{1}$ The Second Affiliated Hospital of Nanjing Medical University, Nanjing 210000, China

Full list of author information is available at the end of the article

\section{Introduction}

As the leading cause of cancer-related death worldwide, colorectal cancer causes over a half million deaths every year [1,2]. Despite considerable progress and therapeutic strategies have been achieved in past decades, the mortality and 5-year survival rate of patients with CRC remain unsatisfactory [3]. Therefore, it is of great importance for us to understand the molecular mechanisms underlying CRC progression and identification of therapeutic biomarkers for improving the CRC patient prognosis and treatment.

Long non-coding RNAs (lncRNAs) are defined as a novel class of RNA molecules of length more than 200 nucleotides with narrow protein coding functions $[4,5]$. Recently, a growing body of research has revealed that 
lncRNAs have been implicated in the tumorigenicity of multiple cancers, including CRC [6-8]. Dysregulated lncRNAs have been demonstrated to work as oncogenes or tumor suppressors through affecting a wide spectrum of biological activities, such as cell growth, invasion, metastasis and autophagy $[9,10]$. For example, Wu et al. suggests that lncRNA TLN2-4 suppresses metastasis and is associated with patient survival in gastric cancer [11]. LncRNA SNHG1 has been identified functioning as a ceRNA to antagonize the effect of miR-145a-5p on the down-regulation of NUAK1 in nasopharyngeal carcinoma cell [12]. Besides, lncRNA PVT1 regulates ovarian cancer cell proliferation by increasing SOX2 expression [13]. However, to the best of our knowledge, the biological functions and the roles of long intergenic non-protein coding RNA 342 (LINC00342) in CRC hadn't been reported yet.

MiRNAs are a class of small noncoding RNAs with a length of about 20-24 nucleotides. An increasing number of reports unveil that miRNAs play a key part in modulating the expression of diverse genes participating in the onset and progression of numerous cancers via regulating mRNA expression [14-16]. Previous studies have suggested that miR-223-3p promotes cell proliferation and invasion by targeting Arid1a in gastric cancer [17]. It is extensively accepted that miR-96 enhances the cervical cancer cell proliferation by targeting FOXO1 [18]. Additionally, miR-29a inhibits cell proliferation and migration by targeting the CDC42/PAK1 signaling pathway in cervical cancer [19]. Long et al. reports that miR-4319 suppresses colorectal cancer progression by targeting ABTB1 [20].

In this study, we demonstrated that LINC00342 was upregulated in CRC tissues and cells. Further study indicated that LINC00342 exerted its carcinogenic effects on the competing endogenous RNA (ceRNA) manner via miR-19a-3p/aminopeptidase like 1 (NPEPL1) axis. Therefore, our study was designed to investigate the interaction among LINC00342, miR-19a-3p and NPEPL1 in the regulation of $\mathrm{CRC}$ progression.

\section{Materials and methods}

\section{Tissues}

Fifty paired tumor tissues and adjacent non-tumor tissues were obtained from CRC patients during their hospitalization in our hospital between July 2016 and January 2018. The expressions of LINC00342 and miR19a-3p were detected by real-time PCR.

\section{Cell lines}

HCT-8, SW480, HT-29 and DLD-1, four human CRC cell lines and human colonic epithelial NCM460 cells were purchased from American Type Culture Collection
(ATCC, Manassas, VA, USA). Cells were incubated in DMEM media supplemented with $10 \%$ fetal bovine serum (Invitrogen, Carlsbad, CA, USA) with $5 \% \mathrm{CO}_{2}$ at $37^{\circ} \mathrm{C}$.

\section{Fluorescence in situ hybridization (FISH)}

The slides of SW480 and HT-29 cells were placed in a 24-well plate at a density of $5 \times 10^{3}$ cells/well, and cultured for $24 \mathrm{~h}$. After washing with PBS, cells were fixed in $4 \%$ paraformaldehyde for $20 \mathrm{~min}$, permeabilized by $0.5 \%$ Trition X-100 in PBS and digested with protein K. Then the hybridization was performed overnight at $37^{\circ} \mathrm{C}$. The slides were finally stained with DAPI and observed under confocal laser microscopy (Zeiss, New York, NY, USA).

\section{Cell transfection}

SW480 and HT-29 cells were transfected with empty vector, pcDNA3.1-LINC0342, mimic NC or miR-19a-3p mimic, synthesized by GenePharma Co., Ltd. (Shanghai, China) using Lipofectamine 2000 (Invitrogen, Carlsbad, CA, USA) following the manufacturer's instructions, or were infected with lentivirus containing shRNA targeting LINC00342 or NPEPL1 or green fluorescent protein (GFP). Transfection efficiency was evaluated by real-time PCR and green fluorescence microscopy.

\section{Luciferase reporter assay}

SW480 and HT-29 cells were co-transfected with the wild/mutated types of LINC00342 or NPEPL1 promoter reporters and miR-19a-3p mimic/mimic NC or pcDNA3.1-LINC00342. Luciferase assays were performed after 48-h transfection.

\section{RNA immunoprecipitation (RIP)}

RIP assay was performed using the Immunoprecipitation Kit (Millipore, Billerica, MA, USA) according to the manufacturer's instructions. Cell extracts of SW480 and HT-29 cells were incubated with protein A/G sepharose beads at $4{ }^{\circ} \mathrm{C}$. Immunoprecipitated RNAs were subjected to real-time PCR.

\section{Cell proliferation}

Cell proliferation was assessed using CCK-8 method and colony formation assay. For CCK- 8 assay, cells were seeded into 96-well plates at a density of $2 \times 10^{3}$ cells/ well, and then CCK-8 dyes (Beyotime, Shanghai, China) were added into each group of cells for co-culture 24, 48 and $72 \mathrm{~h}$ at $37{ }^{\circ} \mathrm{C}$. Then, the optical density (OD) values were measured. For colony formation assay, the colonies were counted after being stained with $0.5 \%$ crystal violet. 


\section{Migration and invasion assay}

For wound-healing assay, cells were wounded by a sterile micropipette tip, and photographed under a microscope (Olympus, Tokyo, Japan) at $0 \mathrm{~h}$ and $48 \mathrm{~h}$ after wounding. For transwell migration assay, cells were prepared in the upper chamber, while the lower chamber contained culture medium with serum. After 12-h culture, cells at the bottom were visualized under a microscope $(\times 200$ magnification) in 5 randomly selected fields of view after stained with $4 \%$ crystal violet. In the transwell invasion assay, filters coated with matrigel matrix were used.

\section{Real-time PCR analysis}

Total RNA was extracted from tissues or cells using Trizol reagent and reverse-transcribed to cDNA. The quantitative analysis of LINC00342, miR-19a-3p and NPEPL1 expression were respectively normalized to GAPDH, U6 and GAPDH, determined using SYBR Premix Ex Taq (TaKaRa, Dalian, China). The specific primers were as followed: LINC00342 forward, 5'-CGTTCCAATGTG TTGGGT-3'and reverse, 5'-TGGGAGGAGGTTGAG ATG-3'; miR-19a-3p forward, 5'-GGCGGGGAAAGT GTGTCT-3'and reverse, 5'-GTGCAGTCGTGGCGT GTG-3'; NPEPL1 forward, 5'-TCAGCCACACCCCAG ATGGA- $3^{\prime}$ and reverse: 5'-AGCCAAGCAGAACAC AGCGT-3'; GAPDH forward, 5'-ACGGATTTGGTC GTATTGGGCG- $3^{\prime}$ and reverse, $5^{\prime}$-CTCCTGGAAGAT GGTGATGG- $3^{\prime}$ and U6 forward, $5^{\prime}$-CTCGCTTCGGCA GCACA- $3^{\prime}$ and reverse, $5^{\prime}$-AACGCTTCACGAATTTGC GT-3'.

\section{Western blot}

After protein quantification, indicated antibodies (1:1000 dilution; Cell Signaling Technology) and horseradish peroxidase-conjugated secondary antibodies were applied for incubation. The target proteins were visualized using ECL system (7Sea PharmTech, Shanghai, China).

\section{Tumor xenograft model}

$\mathrm{BALB} / \mathrm{c}$ nude mice were purchased from Beijing Vitalriver Experimental Animal Technology Co., Ltd. All animal procedures were approved by Animal Care Committee of the Second Affiliated Hospital of Nanjing Medical University. HT-29 cells $\left(1 \times 10^{6}\right)$ stably transfected with sh-LINC00342 or sh-NC were subcutaneously injected into nude mice. The experimental mice were routinely monitored and sacrificed on day 25 by cervical dislocation and their tumors were collected. Tumor volume was measured every 5 days and was calculated by the following formula: volume $\left(\mathrm{mm}^{3}\right)=\left(\right.$ length $\times$ width $\left.{ }^{2}\right) / 2$.

\section{Hematoxylin and eosin (H\&E) staining}

The tumor tissues were fixed in $4 \%$ paraformaldehyde and then embedded in paraffin. The sections were stained with $H \& E$. At least three different sections of tumor tissues were examined for each group using a microscope to assess the histopathological alterations.

\section{Immunohistochemistry (IHC) assay}

Experimental method was as described above [8]. Briefly, lung samples were fixed and cut into $4 \mu \mathrm{m}$ thick sections, dried, deparaffinized, and dehydrated in a graded ethanol series, and finally incubated with $\mathrm{H}_{2} \mathrm{O}_{2}$. Blocked with bovine serum, sections were incubated overnight with monoclonal antibodies (1: 1000 dilutions) of Ki67, E-cadherin and Vimentin. The secondary antibody (1: 500 dilutions) was used to incubate the sections, and then the sections were incubated with horseradish peroxidase-conjugated streptavidin. A 3, $3^{\prime}$-diaminobenzidine (DAB) substrate kit was used, and slides were counterstained with hematoxylin. The section was visualized under a light microscope (Olympus Corporation, Tokyo, Japan).

\section{Statistical analysis}

Data were expressed as mean \pm standard deviation (SD). Comparisons between groups were assessed by two-sided Student's t test or analysis of variance using the SPSS 22.0 (IBM, Armonk, NY, USA). $P<0.05$ was considered statistically significant.

\section{Results \\ LINC00342 is significantly up-regulated in CRC}

Using real-time PCR analysis, the increased expression of LINC00342 was observed in CRC tumor tissues and cell lines $(P<0.01$, Fig. $1 \mathrm{a}, \mathrm{b})$. Analyzed by RNA-FISH, LINC00342 was distributed in the cytoplasm of CRC cells (Fig. 1c).

\section{LINC00342 requires for CRC cell proliferation, migration and invasion}

To investigate the biological functions of LINC00342 in CRC cells, specific shRNA was used to knock down LINC00342 expression in SW480 and HT-29 cells, and real-time PCR (Fig. 2a) and green fluorescence microscopy (Fig. 2b) were performed to confirm the transfection efficiency. The results showed that the cell viability $(P<0.01$, Fig. $2 c)$ and colony formation abilities $(P<0.01$, Fig. 2 d) were inhibited by LINC00342 silencing. The wound healing assay and the transwell assays indicated that suppression of LINC00342 expression significantly inhibited cell migration and invasion $(P<0.01$, Fig. 2e, f). Moreover, the protein levels 

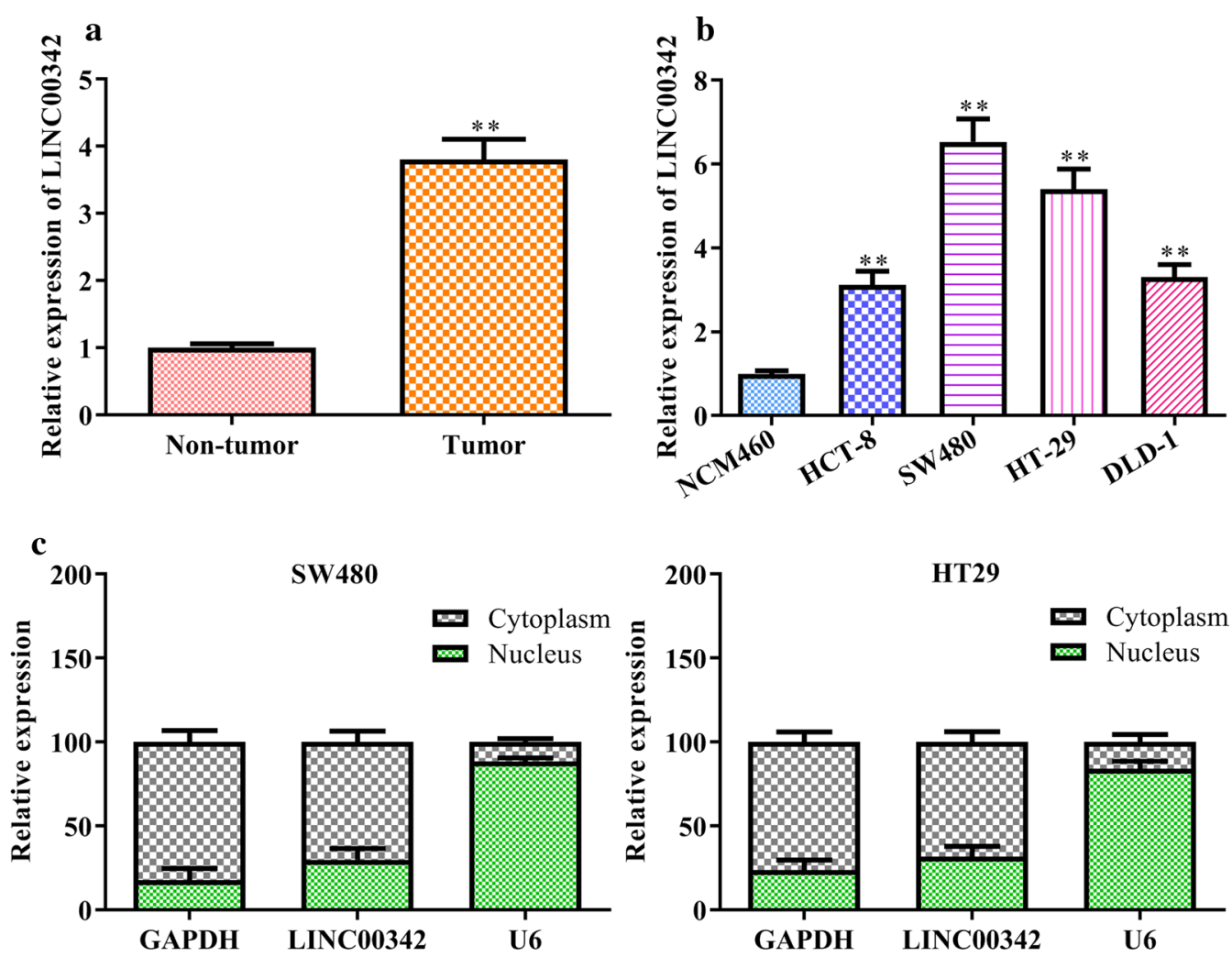

Fig. 1 LINC00342 is significantly upregulated in CRC tissues and cells. a Expression of LINC00342 in non-tumor tissues and CRC tumor tissues determined by real-time PCR; $\boldsymbol{b}$ expression of LINC00342 in colonic epithelial NCM460 cells and four CRC cells (HCT-8, SW480, HT-29 and DLD-1); $\mathbf{c}$ subcellular fractionation assay of LINC00342 in SW480 and HT-29 cells, analyzed by RNA-FISH. ${ }^{* * P}<0.05$ vs. the non-tumor group or NCM460 cells

of E-cadherin were largely increased, while Vimentin expression was observably reduced after LINC00342 silencing $(P<0.01$, Fig. $2 \mathrm{~g})$.

MiR-19a-3p is down-regulated in CRC and negatively regulated by LINC00342.

As shown in Fig. 3a, b, miR-19a-3p was lowly expressed in CRC tissues and cells. Furthermore, miR-19a-3p was predicted as a target of LINC00342 by DIANA tools (http://carolina.imis.athena-innov ation.gr/) (Fig. 3c). Then, dual-luciferase reporter assay demonstrated that miR-19a-3p overexpression after transfection with miR-19a-3p mimic $(P<0.01$, Fig. 3d) significantly reduced the relative luciferase activity of LINC00342-WT in SW480 and HT-29 cells $(P<0.01$, Fig. 3 e). RIP assay indicated that LINC00342 and miR-19a-3p were evidently enriched in Ago2 immunoprecipitates in contrast to IgG immunoprecipitates $(P<0.01$, Fig. $3 f)$. In addition, knockdown of LINC00342 promoted the miR-19a-3p expression in CRC cells $(P<0.01$, Fig. $3 g)$.

\section{MiR-19a-3p inhibits CRC cell proliferation, migration} and invasion

We explored the biological function of miR-19a-3p in CRC. Ectopic expression of miR-19a-3p memorably reduced cell proliferation $(P<0.01$, Fig. $4 \mathrm{a})$ and colony formation $(P<0.01$, Fig. 4b) in SW480 and HT-29 cells. The migration and invasion abilities of CRC cells were diminished after up-regulation of miR-19a-3p $(P<0.01$, Fig. 4c, d). Thereafter, overexpression of miR-19a-3p significantly increased E-cadherin expression but suppressed the protein levels of Vimentin $(P<0.01$, Fig. $4 \mathrm{e})$.

\section{LINC00342 functions as a ceRNA of NPEPL1 by sponging miR-19a-3p}

Predicted by TargetScan (http://www.targetscan.org), NPEPL1 was selected as a candidate target due to the carcinogenic role of miR-19a-3p in several cancers (Fig. 5a). Luciferase assay in SW480 and HT-29 cells confirmed that overexpression of miR-19a-3p strikingly reduced the luciferase activity of NPEPL1-WT $(P<0.01$, Fig. 5b), 

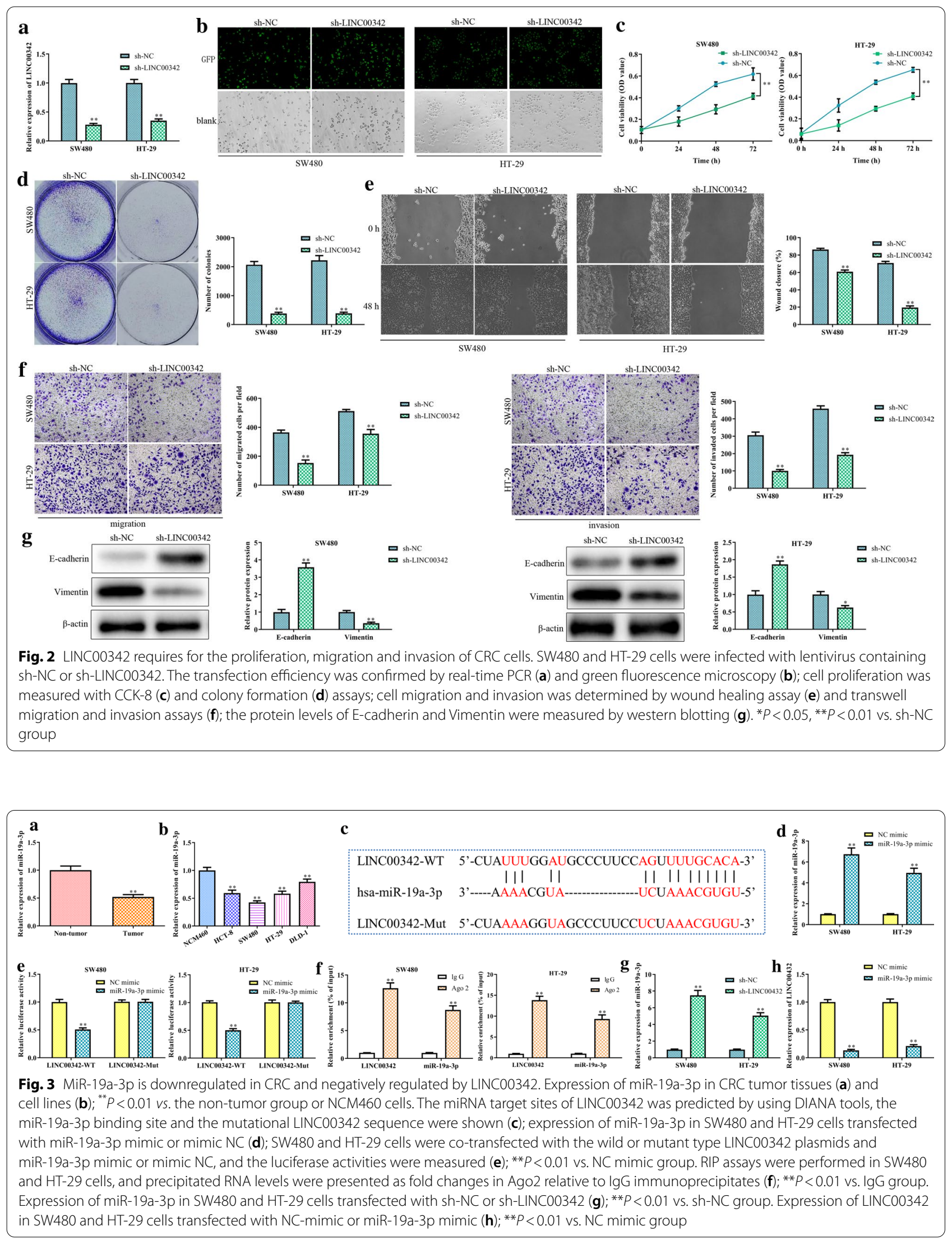


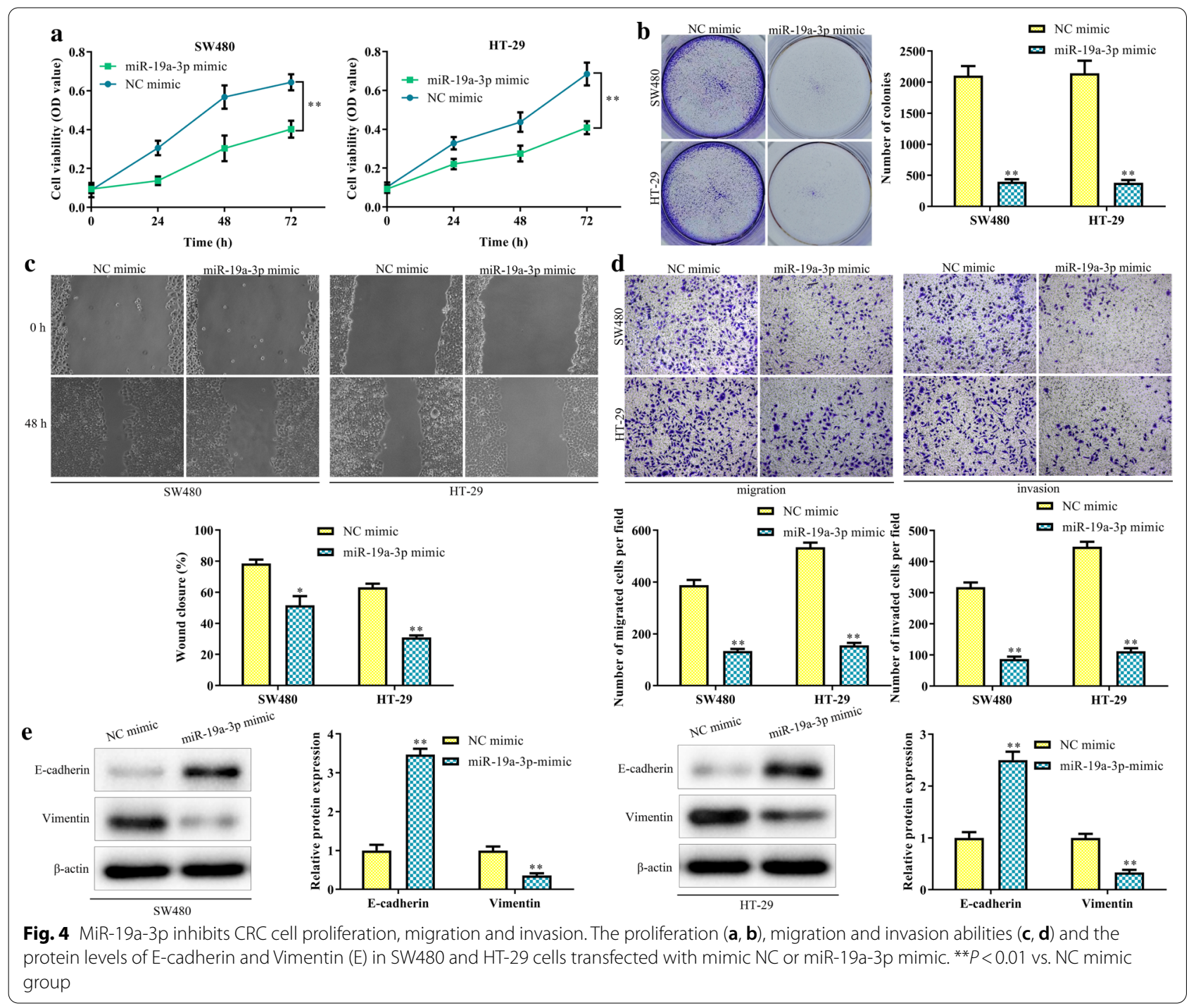

indicating that miR-19a-3p directly bound to NPEPL1 in CRC cells, which was verified by RIP experiments $(P<0.01$, Fig. $5 \mathrm{c})$. Moreover, we found that the mRNA and protein expression of NPEPL1 was reduced by miR-19a-3p mimic in SW480 and HT-29 cells $(P<0.01$, Fig. $5 \mathrm{~d}, \mathrm{e})$. Luciferase assays showed that the reduced luciferase activity of NPEPL1-WT, induced by overexpression of miR-19a-3p was eliminated by LINC00342 $(P<0.01$, Fig. 5f $)$. The downregulated NPEPL1 expression at mRNA and protein levels were observed after knockdown of endogenous LINC00342 $(P<0.01$, Fig. $5 \mathrm{~g}, \mathrm{~h})$.

\section{Knockdown of NPEPL1 rescues the carcinogenesis of LINC00342 on CRC progression}

Rescue experiments were performed in SW480 cells by transfection with LINC00342 and/or sh-NPEPL1 to investigate whether LINC00342-induced carcinogenesis were mediated by miR-19a-3p/NPEPL1 axis in CRC cells, (Fig. 6a). The proliferation $(P<0.01$, Fig. 6b, c) migration and invasion capacities $(P<0.01$, Fig. $6 \mathrm{~d}$, e) were elevated by LINC00342 overexpression, but repressed by NPEPL1 down-regulation. Additionally, restoration of LINC00342 expression markedly inhibited E-cadherin expression, but increased Vimentin levels, whereas NPEPL1 silencing led to an opposite effect. However, the pro-tumor effects of LINC00342 were reversed by NPEPL1 knockdown $(P<0.01$, Fig. 6f $)$.

\section{Silencing LINC00342 inhibits tumorigenesis of CRC cells through in vivo}

Xenograft nude mouse models were established in order to elucidate the role of LINC00342 in CRC 


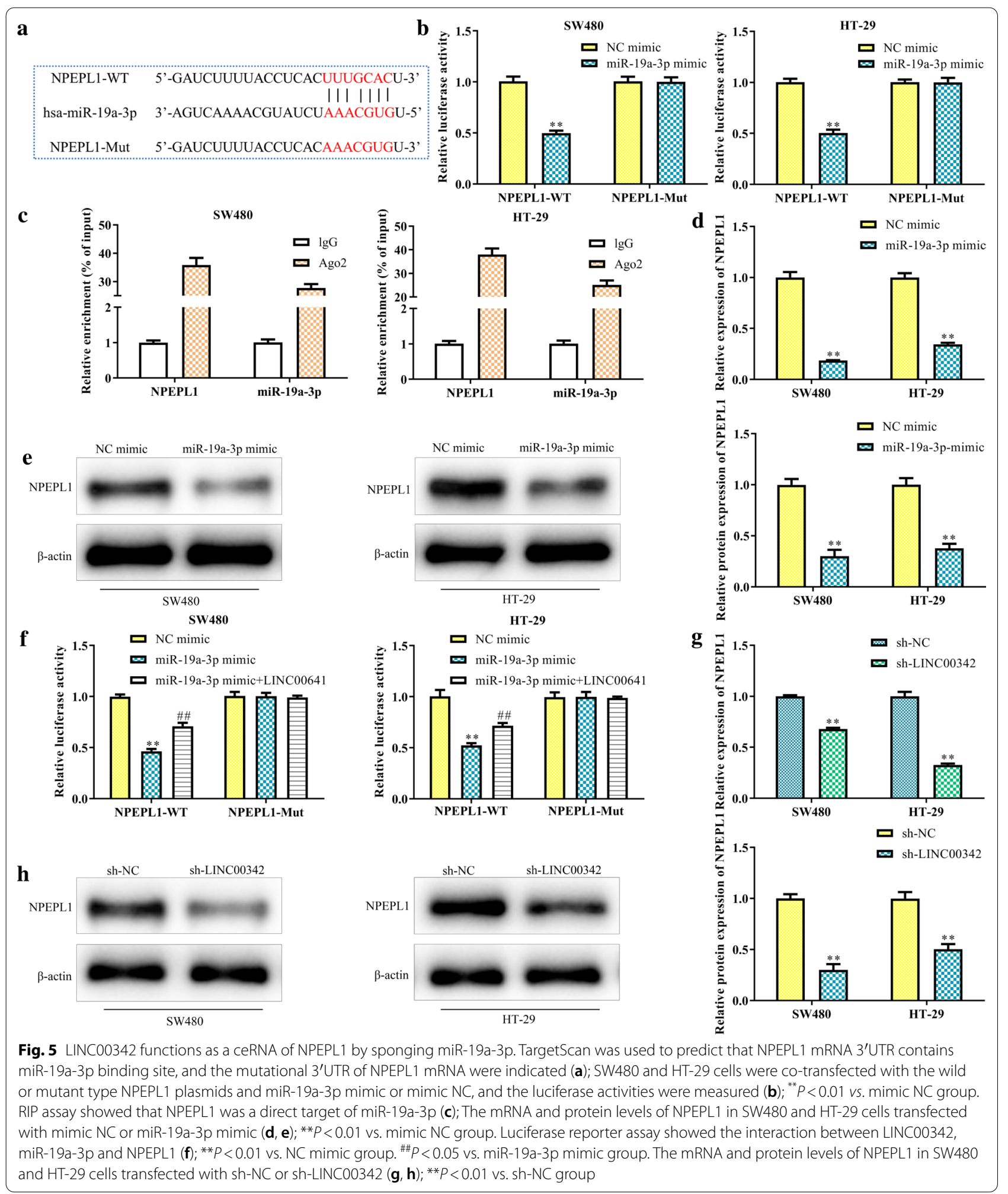

in vivo. The results showed that LINC00342 knockdown exhibited lower tumor growth compared with sh-NC group $(P<0.001$, Fig. $7 \mathrm{a}, \mathrm{b})$. Moreover, compared with the sh-NC, tumor weight was reduced by LINC00342 silencing $(P<0.01$, Fig. $7 \mathrm{c})$. In addition, with the analysis of histological and IHC in tumor 


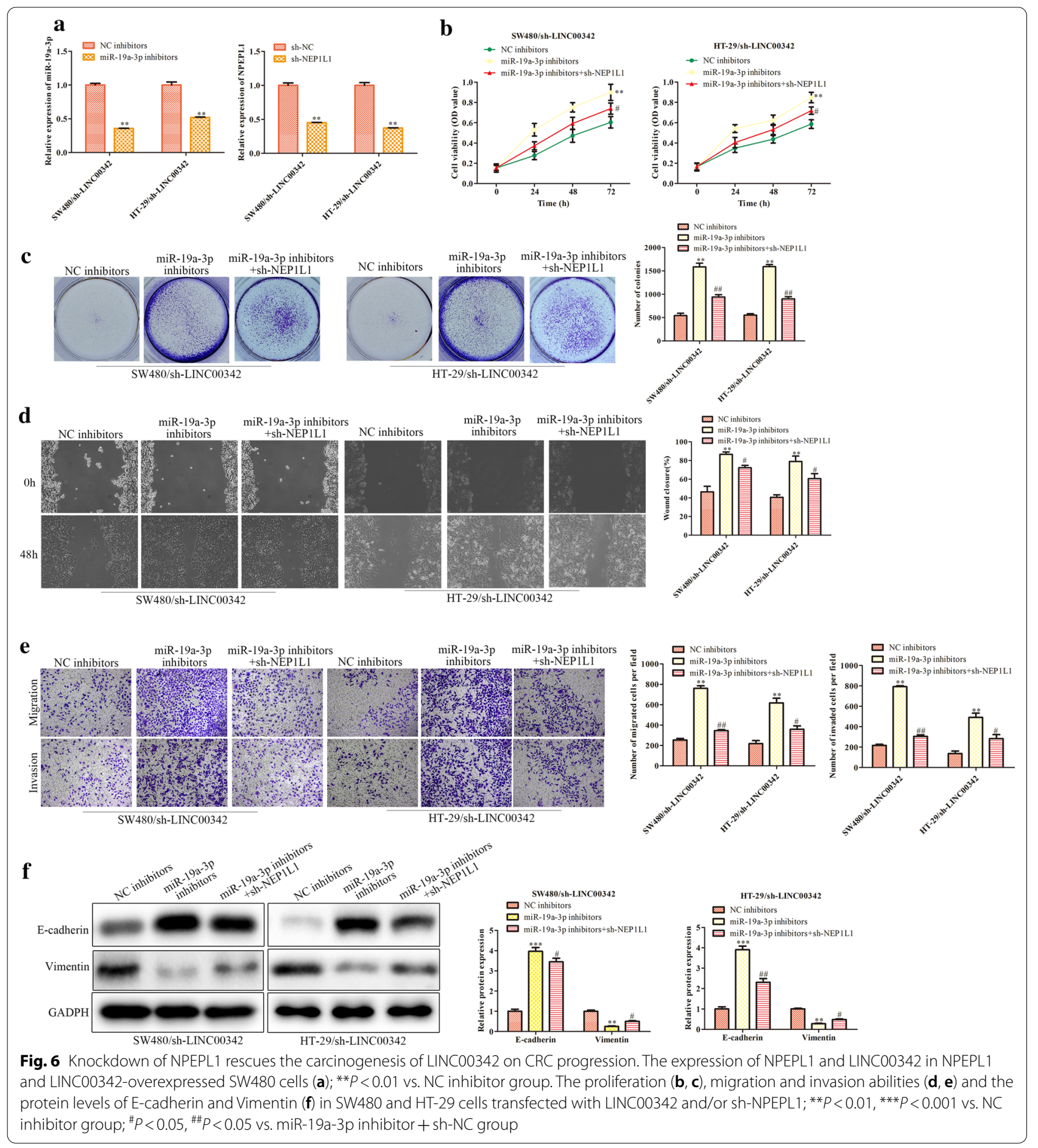

sections, the expression levels of Ki67 and vimentin were higher, while the E-cadherin expression level was lower in the sh-LINC00342 group than the sh-NC group $(P<0.01$, Fig. $7 \mathrm{~d}-\mathrm{f})$. Hence, these findings indicated that knockdown of LINC00342 inhibited the tumor growth in vivo. 

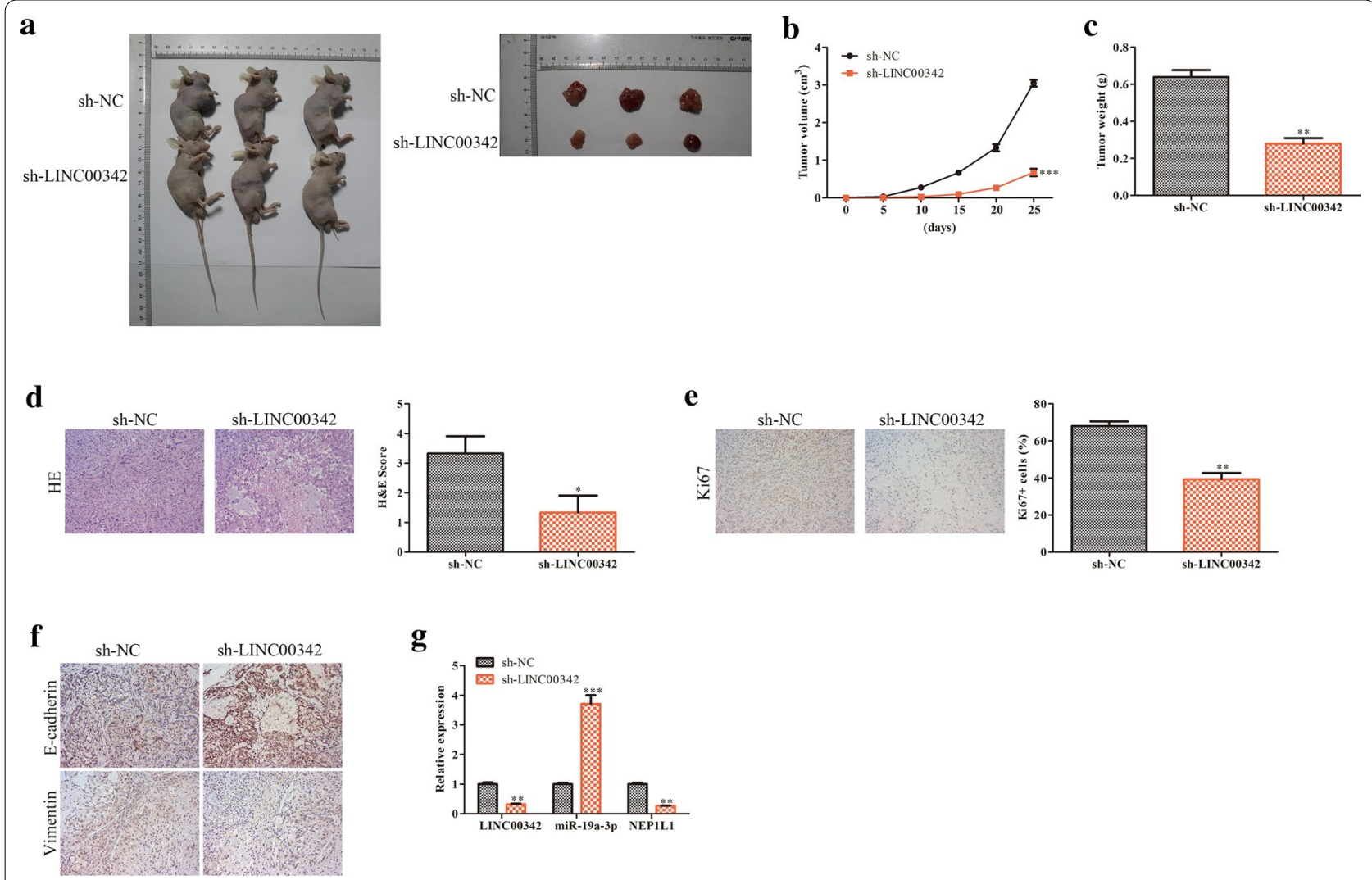

Fig. 7 Silencing LINC00342 inhibits tumorigenesis of CRC cells through in vivo. Representative images of nude mice and tumors (a); the growth curves of tumors derived from HT-29 cells transfected with sh-LINC00342 or si-NC (b); the average weight of tumors (c); the analysis of histological in tumor sections (d); immunohistochemistry assay was conduct to assess the expression levels of Ki67, E-cadherin and vimentin. The photographs were taken at the magnification of $\times 200(E, F) .{ }^{*} P<0.05,{ }^{* *} P<0.01,{ }^{* * *} P<0.001$ vs. sh-NC group

\section{Discussion}

The critical functions of IncRNAs have been reported in CRC development. For instance, MALAT1 expression is correlated with the maintenance of CRC stem cell properties [21]. LncRNA PCAT6 modulates 5-fluorouracil chemoresistance in CRC cells through a miR-204/ HMGA2 pathway [22]. LncRNA HOTAIR expression is associated with the aggressive biological behaviors of CRC cells [23]. In our research, we found that LINC00342 was up-regulated in CRC. Functional analysis revealed that LINC00342 silencing inhibited proliferation, migration and invasion in vitro and in vivo in CRC. Moreover, LINC00342 functioned as a tumor promoter by sponging miR-19a-3p and releasing NPEPL1. Taken together, our study illuminated that a LINC00342/miR19a-3p/NPEPL1 axis existed in CRC.

LINC00342, a novel lncRNA located on chromosome $2 \mathrm{q} 11.1$, is up-regulated in non-small cell lung cancer (NSCLC) and infantile hemangiomas [24, 25]. High expression of LINC00342 predicts a poor prognosis in NSCLC [26]. Knockdown of LINC00342 inhibits NSCLC cell proliferation, migration and invasion [27].
However, the expression and function of LINC00342 remained unknown in CRC. Herein, LINC00342 was highly expressed in CRC tissues and cells. Our study further demonstrated that LINC00342 induced the proliferation and metastasis of CRC cells and tumor growth. These findings suggested that LINC00342 acted as an oncogene in CRC.

Increasing evidence shows a novel regulatory mechanism that lncRNAs serve as miRNA sponges at a post-transcriptional level in the occurrence and development of various tumors. For example, $\mathrm{Xu}$ et al. has reported that lncRNA TUSC7 functions as a miR-211 decoy in the regulation of $\mathrm{CDK} 6 \mathrm{SH} 3$ and suppresses the proliferation of CRC cells [28]. Li et al. has exhibited that lncRNA MFI2-AS1 regulates CRC cell proliferation, metastasis, cell cycle distribution, and apoptosis through competitively interacting with miR574-5p to upregulate MYCBP expression [29]. Shi et al. finds that ZNFX1-AS1 serves as a ceRNA to upregulate EZH2 expression by sponging miR-144 to promote CRC progression [30]. In our study, we discovered that LINC00342 was involved in the ceRNA regulatory 
network and functioned as an endogenous miRNA sponge to bind to miR-19a-3p and regulated its function. In line with our finding, Chen et al. reports that ectopic LINC00342 expression facilitates NSCLC cell proliferation, migration, and invasion by sponging miR203a-3p [27].

MiR-19a-3p functioned as an oncogene or a tumor suppressor in various cancers. For example, miR-19a-3p is distinctly increased in hepatocellular carcinoma (HCC), and promotes $\mathrm{HCC}$ cell metastasis and chemoresistance [31]. In contrast, miR-19a-3p expression is reduced in osteosarcoma, and silencing miR-19a-3p suppresses osteosarcoma cell proliferation and induces apoptosis [32]. In addition, miR-19a-3p is reported to be down-regulated and to further inhibit the angiogenesis of CRC cells [33]. However, how miR-19a-3p was controlled in CRC remained unclear.

In this study, enforced overexpression of miR-19a-3p reduced the capabilities of proliferation, migration and invasion of CRC cells, suggesting that miR-19a-3p might play a tumor-suppressive role in CRC. Bioinformatics prediction and luciferase reporter assays confirmed that a direct binding existed between LINC00342 and miR19a-3p. In addition, the oncogene NPEPL1 was demonstrated to be a direct target of miR-19a-3p, which was consistent with the previous research [34]. Functional analyses revealed that the overexpression of LINC00342 partly overturned the tumor suppressive effects of NPEPL1 knockdown in CRC, indicating that LINC00342 accelerated CRC progression, at least in part, through regulating the expression of NPEPL1 by serving as a sponge of miR-19a-3p.

\section{Conclusion}

In summary, our study for the first time revealed a novel LINC00342-miR-19a-3p-NPEPL1 regulatory network in the pathogenesis and development of CRC: LINC00342 promoted CRC progression by competitively binding miR-19a-3p with NPEPL1. The limitation of this study was that the exploration on the mechanism of LINC00342 was not deep enough. We would further investigate the mechanism and network of LINC00342 in the future work.

\section{Acknowledgements}

Not applicable.

\section{Authors' contributions}

Conceived and designed the study: PS, GJ. Performed the literature search and data extraction: PS, LQ, JW, QD, CZ, RX, HW, GJ. Analyzed the data: PS, GJ. Drafted the manuscript: PS, GJ. All authors read and approved the final manuscript.

\section{Funding}

The Science and Technology Development Foundation of Nanjing Medical University of China (No. 2016NJMUZD03).

\section{Availability of data and materials}

The raw data supporting the conclusions of this manuscript will be made available by the authors, without undue reservation, to any qualified researcher.

\section{Ethics approval and consent to participate}

Our project was approved by Research Ethics Committee of The Second Affiliated Hospital of Nanjing Medical University (approval number: 2015KY(051). And our study obtained the informed consent of all patients.

\section{Consent for publication}

All the authors agree to the publication of this manuscript.

\section{Competing interests}

The authors state that there is no conflict of interest.

\section{Author details}

${ }^{1}$ The Second Affiliated Hospital of Nanjing Medical University, Nanjing 210000, China. ${ }^{2}$ The Affiliated Huaian No.1 People's Hospital of Nanjing Medical University, Huai'an 223300, China.

Received: 15 June 2020 Accepted: 7 December 2020

Published online: 15 February 2021

\section{References}

1. Marmol I, Sanchez-de-Diego C, Pradilla Dieste A, Cerrada E, Rodriguez Yoldi MJ. Colorectal carcinoma: a general overview and future perspectives in colorectal cancer. Int J Mol Sci. 2017;18(1):197.

2. Zamani M, Hosseini SV, Mokarram P. Epigenetic biomarkers in colorectal cancer: premises and prospects. Biomarkers. 2018;23(2):105-14.

3. Goldstein DA, Zeichner SB, Bartnik CM, Neustadter E, Flowers CR. Metastatic colorectal cancer: a systematic review of the value of current therapies. Clin Colorectal Cancer. 2016;15(1):1-6.

4. Jarroux J, Morillon A, Pinskaya M. History, discovery, and classification of IncRNAs. Adv Exp Med Biol. 2017;1008:1-46.

5. Kopp F, Mendell JT. Functional classification and experimental dissection of long noncoding RNAs. Cell. 2018;172(3):393-407.

6. Luo Y, Chen JJ, Lv Q, Qin J, Huang YZ, Yu MH, Zhong M. Long non-coding RNA NEAT1 promotes colorectal cancer progression by competitively binding miR-34a with SIRT1 and enhancing the Wnt/beta-catenin signaling pathway. Cancer Lett. 2019;440-441:11-22.

7. Yang MH, Zhao L, Wang L, Ou-Yang W, Hu SS, Li WL, Ai ML, Wang YQ, Han Y, Li TT, Ding YQ, Wang S. Nuclear IncRNA HOXD-AS1 suppresses colorectal carcinoma growth and metastasis via inhibiting HOXD3-induced integrin beta3 transcriptional activating and MAPK/AKT signalling. Mol Cancer. 2019;18(1):31.

8. Wang $Y Q$, Jiang $D M$, Hu SS, Zhao L, Wang L, Yang MH, Ai ML, Jiang HJ, Han Y, Ding YQ, Wang S. SATB2-AS1 suppresses colorectal carcinoma aggressiveness by inhibiting SATB2-dependent snail transcription and epithelial-mesenchymal transition. Can Res. 2019;79(14):3542-56.

9. Qi G, Li L. Long non-coding RNA PVT1 contributes to cell growth and metastasis in non-small-cell lung cancer by regulating miR-361-3p/SOX9 axis and activating Wnt/ $\beta$-catenin signaling pathway. Biomed Pharmacother. 2020;126:110100.

10. Chen Y, Gao H, Li Y. Inhibition of LncRNA FOXD3-AS1 suppresses the aggressive biological behaviors of thyroid cancer via elevating miR296-5p and inactivating TGF- $\beta 1 /$ Smads signaling pathway. Mol Cell Endocrinol. 2019;500:110634.

11. Wu Y, Hao N, Wang S, Yang X, Xiao Y, Yang H, Yang S, Li B. Long noncoding RNA Lnc-TLN2-4:1 suppresses gastric cancer metastasis and is associated with patient survival. J Oncol. 2020;2020:1-8.

12. Lan $X$, Liu X. LncRNA SNHG1 functions as a ceRNA to antagonize the effect of miR-145a-5p on the down-regulation of NUAK1 in nasopharyngeal carcinoma cell. J Cell Mol Med. 2019;23(4):2351-61. 
13. Zou M-F, Ling J, et al. Long non-coding RNA PVT1 functions as an oncogene in ovarian cancer via upregulating SOX2. Eur Rev Med Pharmacol Sci. 2018;22(21):7183-8.

14. Peng X, Zhang Y, Gao J, Cai C. MiR-1258 promotes the apoptosis of cervical cancer cells by regulating the E2F1/P53 signaling pathway. Exp Mol Pathol. 2020;114:104368.

15. Song Y, Guo Q, Gao S, Hua K. miR-454-3p promotes proliferation and induces apoptosis in human cervical cancer cells by targeting TRIM3. Biochem Biophys Res Commun. 2019;516(3):872-9.

16. Yilin L, Zhihua C, Suyong L, Yan Z, Yisu L, Ji G, Shaoqin C. MiR-202 inhibits the proliferation and invasion of colorectal cancer by targeting UHRF1. Acta Biochim et Biophys Sin. 2019;51:598-606.

17. Yiping Z, Kai L, Liang Y, Yang H, Lu W, Lili S. miR-223-3p promotes cell proliferation and invasion by targeting Arid1a in gastric cancer. Acta Biochim et Biophys Sin. 2020;52:150-9.

18. Yang L, Liu L, Zhang X, Zhu Y, Li L, Wang B, Liu Y, Ren C. Research, Practice, miR-96 enhances the proliferation of cervical cancer cells by targeting FOXO1. Pathol Res Pract. 2020;216(4):152854.

19. Chen R, Zhang L. MiR-29a inhibits cell proliferation and migration by targeting the CDC42/PAK1 signaling pathway in cervical cancer. Anticancer Drugs. 2019;30:579-87.

20. Huang L, Zhang Y, Li Z, Zhao X, Xi Z, Chen H, Shi H, Xin T, Shen R, Wang T. MiR-4319 suppresses colorectal cancer progression by targeting ABTB1. United Eur Gastroenterol J. 2019;7:517-28.

21. Tang D, Yang Z, Long F, Luo L, Yang B, Zhu R, Sang X, Cao G, Wang K. Long noncoding RNA MALAT1 mediates stem cell-like properties in human colorectal cancer cells by regulating miR-20b-5p/Oct4 axis. J Cell Physiol. 2019;234(11):20816-28.

22. Wu H, Zou Q, He H, Liang Y, Lei M, Zhou Q, Fan D, Shen L. Long noncoding RNA PCAT6 targets miR-204 to modulate the chemoresistance of colorectal cancer cells to 5-fluorouracil-based treatment through HMGA2 signaling. Cancer Med. 2019;8(5):2484-95.

23. Pan S, Liu Y, Liu Q, Xiao Y, Liu B, Ren X, Qi X, Zhou H, Zeng C, Jia L. HOTAIR/ miR-326/FUT6 axis facilitates colorectal cancer progression through regulating fucosylation of CD44 via PI3K/AKT/mTOR pathway. Biochimica et biophysica acta Molecular Cell Res. 2019;1866(5):750-60.

24. Wang L, Chen Z, An L, Wang Y, Zhang Z, Guo Y, Liu C. Analysis of long non-coding RNA expression profiles in non-small cell lung cancer. Cell Physiol Biochem. 2016;38(6):2389-400.

25. Liu Z, Kang Z, Dai Y, Zheng H, Wang Y. Long noncoding RNA LINC00342 promotes growth of infantile hemangioma by sponging miR-3619-5p from HDGF. Am J Physiol Heart Circ Physiol. 2019;317(4):H830-9.
26. Tang H, Zhao L, Li M, Li T, Hao Y. Investigation of LINC00342 as a poor prognostic biomarker for human patients with non-small cell lung cancer. J Cell Biochem. 2019;120(4):5055-61.

27. Chen QF, Kong JL, Zou SC, Gao H, Wang F, Qin SM, Wang W. LncRNA LINC00342 regulated cell growth and metastasis in non-small cell lung cancer via targeting miR-203a-3p. Eur Rev Med Pharmacol Sci. 2019;23(17):7408-18.

28. Xu J, Zhang R, Zhao J. The novel long noncoding RNA TUSC7 inhibits proliferation by sponging MiR-211 in colorectal cancer. Cell Physiolo Biochem. 2017;41(2):635-44.

29. Li C, Tan F, Pei Q, Zhou Z, Zhou Y, Zhang L, Wang D, Pei H. Noncoding RNA MFI2-AS1 promotes colorectal cancer cell proliferation, migration and invasion through miR-574-5p/MYCBP axis. Cell Prolif. 2019;52(4):e12632.

30. Shi L, Hong X, Ba L, He X, Xiong Y, Ding Q, Yang S, Peng G. Long noncoding RNA ZNFX1-AS1 promotes the tumor progression and metastasis of colorectal cancer by acting as a competing endogenous RNA of miR144 to regulate EZH2 expression. Cell Death Dis. 2019:10(3):150.

31. Jiang XM, Yu XN, Liu TT, Zhu HR, Shi X, Bilegsaikhan E, Guo HY, Song GQ, Weng SQ, Huang XX, Dong L, Janssen HLA, Shen XZ, Zhu JM. microRNA19a-3p promotes tumor metastasis and chemoresistance through the PTEN/Akt pathway in hepatocellular carcinoma. Biomed Pharmacother Biomed Pharmacother. 2018;105:1147-54.

32. Zhang B, Liu Y, Zhang J. Silencing of miR-19a-3p enhances osteosarcoma cells chemosensitivity by elevating the expression of tumor suppressor PTEN. Oncol Lett. 2019;17(1):414-21.

33. Chen M, Lin M, Wang X. Overexpression of miR-19a inhibits colorectal cancer angiogenesis by suppressing KRAS expression. Oncol Rep. 2018;39(2):619-26.

34. Ouchida M, Kanzaki H, Ito S, Hanafusa H, Jitsumori Y, Tamaru S, Shimizu K. Novel direct targets of miR-19a identified in breast cancer cells by a quantitative proteomic approach. PLoS ONE. 2012;7(8):e44095.

\section{Publisher's Note}

Springer Nature remains neutral with regard to jurisdictional claims in published maps and institutional affiliations.
Ready to submit your research? Choose BMC and benefit from:

- fast, convenient online submission

- thorough peer review by experienced researchers in your field

- rapid publication on acceptance

- support for research data, including large and complex data types

- gold Open Access which fosters wider collaboration and increased citations

- maximum visibility for your research: over $100 \mathrm{M}$ website views per year

At BMC, research is always in progress.

Learn more biomedcentral.com/submissions 